\title{
CONCERNING INTEGRALS
}

PASQUALE PORCELLI

1. Introduction. R. E. Lane [2] has given the following definition of an integral on the interval $[a, b]$ of the function $f$ with respect to the function $g$. If $D$ is an ordered subdivision $\left\{x_{i}\right\}_{i=0}^{n+1}$ of the interval $[a, b], \sum_{D}(f, g)$ denotes the sum

$$
\sum_{i=1}^{n+1} \frac{1}{2}\left[f\left(x_{i}\right)+f\left(x_{i-1}\right)\right]\left[g\left(x_{i}\right)-g\left(x_{i-1}\right)\right] .
$$

The statement that $f$ is g-integrable on $[a, b]$ means that there exists a number $J$ such that for each positive number $\epsilon$ there is an ordered subdivision $D$ of $[a, b]$, such that for every refinement $D^{\prime}$ of $D$, $\left|J-\sum_{D^{\prime}}(f, g)\right|<\epsilon$. The number $J$ is the integral on $[a, b]$ of $f$ with respect to $g$, and is denoted by $\int_{a}^{b} f d g$. This integral generalizes the Stieltjes integral and has many of its properties, e.g., is an additive function of intervals and a bilinear function of $(f, g)$; if $f$ is $g$-integrable on $[a, b]$, then $g$ is $f$-integrable on $[a, b]$ and $\int_{a}^{b} g d f=\left.g f\right|_{a} ^{b}$ $-\int_{a}^{b} f d g$. If $g \in B V[a, b]$ and $f$ has only discontinuities of the first kind in $[a, b]$, then $f$ is $g$-integrable on $[a, b]$ and, in particular, if $g$ is a simple step-function, ${ }^{1}$ then

$$
\begin{aligned}
\int_{a}^{b} f d g=\sum_{a<x \leqq b} \frac{f(x)+f(x-)}{2}[g(x)-g(x-)] \\
+\sum_{a \leqq x<b} \frac{f(x+)+f(x)}{2}[g(x+)-g(x)] .
\end{aligned}
$$

Suppose $g$ is a nondecreasing function and $\left\{f_{n}\right\}_{n=1}^{\infty}$ a uniformly bounded sequence of simple step-functions converging to a function $f$ in $[a, b]-S$, where $S$ is a subset of $[a, b]$ of " $g$-length 0. " It is to be expected that the methods of F. Riesz [3] can be applied to the sequence $\left\{\int_{a}^{b} f_{n} d g\right\}_{n=1}^{\infty}$, and its limit used to define a Riesz type integral of $f$ with respect to $g$ on $[a, b]$.

In this paper we have reached the desired result, but by methods which are in some way even more elementary than those of Riesz.

Presented to the Society, September 5, 1953; received by the editors August 3, 1953 and, in revised form, October 14, 1953.

1 The statement that $g$ is a simple step-function means that $g$ is a function on the set of all numbers and if $[a, b]$ is an interval, then there exist a subdivision $a=x_{0}$ $<x_{1}<\cdots<x_{n}=b$ and a sequence $\left\{k_{p}\right\}_{p-1}^{n}$ of numbers, such that $g(x)=k_{p}$ if $k_{p-1}$ $<x<k_{p}$. 
We depend upon approximation to the nondecreasing function $g$ by step-functions, and essentially reduce the question of measure of $S$ to that of the measure of a finite subset of $S$.

2. Outer $g$-length of a number set. Throughout this paper, $g$ denotes a nondecreasing function on the set of all numbers.

If $S$ is a number set, then the statement that $l_{g} S$ is the outer g-length of $S$ means that $l_{g} S$ is the largest number $k$ such that if $G$ is a countable collection of segments covering $S$, then $k \leqq \sum[g(q-)-g(p+)]$, the sum being taken over every segment $(p, q)$ in $G$.

We state here, without proof, some elementary properties of outer g-length.

(i) The outer $g$-length of the interval $[a, b]$ is $g(b+)-g(a-)$.

(ii) The outer $g$-length of the segment $(a, b)$ is $g(b-)-g(a+)$.

(iii) If $Q$ is an open and bounded number set and $\epsilon$ a positive number, then there exists a finite collection $G$ of mutually exclusive intervals, such that ${ }^{2} G^{*}$ is a subset of $Q, l_{g}[a, b]=g(b)-g(a)$ for each interval $[a, b]$ in $G$, and $0 \leqq l_{\theta} Q-l_{\theta} G^{*}<\epsilon$.

(iv) If $S$ and $T$ are bounded and mutually exclusive number sets and $h$ is a nondecreasing simple step-function, then $l_{h}(S+T)=l_{h} S$ $+l_{h} T$.

(v) If each of $S$ and $T$ is a bounded number set, then $l_{g}(S+T)$ $\leqq l_{o} S+l_{g} T$.

THEOREM A. If $S$ is a bounded number set, each of $\epsilon$ and $\delta$ a positive number and $l_{0} S \geqq \delta$, then there exists a nondecreasing simple step-function $h$, such that, for, every number $x,|h(x)-g(x)|<\epsilon$ and $l_{h} S \geqq \delta$.

Proof. Suppose $[a, b]$ is an interval containing $S$. There exists an ordered subdivision $\left\{x_{i}\right\}_{i=0}^{n+1}$ of $[a, b]$ such that if $x \in\left(x_{i-1}, x_{i}\right)$ then $\left|g(x)-g\left(x_{i-1}+\right)\right|<\epsilon / 2$. Suppose $\left\{y_{i}\right\}_{i=1}^{n+1}$ is a sequence of numbers such that $y_{i} \in\left(x_{i-1}, x_{i}\right)$ and $y_{i} \in S$ if $\left(x_{i-1}, x_{i}\right)$ contains a number belonging to $S$. There exists a number $a^{\prime}$ less than $a$, such that if $x \in\left(a^{\prime}, a\right)$ then $|g(x)-g(a-)|<\epsilon / 2$ and a number $b^{\prime}$ greater than $b$, such that if $x \in\left(b, b^{\prime}\right)$ then $|g(x)-g(b+)|<\epsilon / 2$.

There exist a simple step-function $h_{1}$ on the set of all numbers less than or equal to $a^{\prime}$, such that if $x \leqq a^{\prime}$ then $\left|h_{1}(x)-g(x)\right|<\epsilon / 2$, $h_{1}\left(a^{\prime}\right)=g\left(a^{\prime}\right)$, and $h_{1}$ is nondecreasing, and a simple step-function $h_{2}$ on the set of all numbers greater than or equal to $b^{\prime}$, such that if $x \geqq b^{\prime}$ then $\left|h_{2}(x)-g(x)\right|<\epsilon / 2, h_{2}\left(b^{\prime}\right)=g\left(b^{\prime}\right)$, and $h_{2}$ is nondecreasing (cf. [2]).

${ }^{2}$ If $G$ is a collection of sets, then $G^{*}$ denotes the set that is the logical sum of the sets in $G$. 
$h$ denotes the simple step-function defined as follows: if $x \leqq a^{\prime}$, $h(x)=h_{1}(x) ;$ if $x \geqq b^{\prime}, h(x)=h_{2}(x)$; if $a^{\prime}<x<a, h(x)=g\left(a^{\prime}+\right)$; if $b<x<b^{\prime}, h(x)=g\left(b^{\prime}-\right), h\left(x_{i}\right)=g\left(x_{i}\right)$ if $i=0,1, \cdots, n+1$; if $y_{i} \notin S$ and $x_{i-1}<x<x_{i}, h(x)=g\left(y_{i}\right)$; and if $y_{i} \in S, h(x)=g\left(x_{i-1}+\right)$ or $h(x)$ $=g\left(x_{i}-\right)$, according as $x_{i-1}<x<y_{i}$ or $y_{i} \leqq x<x_{i}$, respectively.

$h$ is a nondecreasing simple step-function, and if $x$ is a number, then $|h(x)-g(x)|<\epsilon$.

Suppose $l_{h} S<\delta$.

If $S$ is a subset of $\left\{x_{i}\right\}_{i=0}^{n+1}$ then, inasmuch as $g\left(x_{i}+\right)-g\left(x_{i}-\right)$ $\leqq h\left(x_{i}+\right)-h\left(x_{i}-\right)$, we see that $l_{g} S \leqq l_{h} S<\delta$, contrary to the hypothesis of the theorem. Therefore, there is a subset of $S$ in one of the segments $\left(x_{i-1}, x_{i}\right)$. Suppose $M$ denotes the collection of these segments containing subsets of $S$, and $S_{1}$ is the common part of $S$ and $M^{*}$. If one of the numbers $x_{i}$ belongs to $S$, denote by $S_{2}$ the common part of $S$ and $\left\{x_{i}\right\}_{i=0}^{n+1}$ and suppose $k=l_{h} S_{2}$. If $S=S_{1}$ then $k=0$.

Suppose $\sum_{(p, q) \in M}[g(q-)-g(p+)]<l_{g} S-k$. Then there exists a positive number $t$ such that $\sum_{(p, q) \in M}[g(q-)-g(p+)]+k+t<l_{g} S$. If $S=S_{1}$, so that $k=0$, this implies $l_{g} S<l_{g} S$, an absurdity. If $S \neq S_{1}$, there is a finite collection $H$ of segments covering $S_{2}$ such that $\sum_{(p, q) \in H}[g(q-)-g(p+)]<k+t$, and therefore

$$
l_{g} S \leqq \sum_{(p, q) \in M+H}[g(q-)-g(p+)]<l_{g} S,
$$

an absurdity. Consequently, we see that

$$
k+\sum_{(p, q) \in M}[g(q-)-g(p+)] \geqq l_{g} S \geqq \delta .
$$

If $S=S_{1}$, this states that $l_{h} S \geqq \delta$.

If $S \neq S_{1}$, this states that $l_{h} S_{2}+l_{h} S_{1} \geqq \delta$ so that, by (iv), $l_{h} S \geqq \delta$.

Thus, the supposition $l_{h} S<\delta$ is false, and Theorem A is established.

3. Sequences of simple step-functions. The following theorem is along the lines of a theorem of Egoroff [1].

TheOREM B. If $S$ is a proper subset of the interval $[a, b], l_{g} S=0$, $\left\{h_{n}\right\}_{n=1}^{\infty}$ is a sequence of step-functions such that, for each number $x$ in $[a, b]-S, h_{n}(x) \rightarrow 0$ as $n \rightarrow \infty$, and each of $\epsilon$ and $\delta$ is a positive number, then there exists a subset $T$ of $[a, b]-S$ and a positive integer $N$ such that, for each $x$ in $T$ and each integer $n$ greater than $N,\left|h_{n}(x)\right|<\epsilon$ and $l_{g} T \geqq l_{g}[a, b]-\delta$.

Proof. Suppose there exists a positive number $\epsilon$ and a positive number $\delta$, such that if $N$ is a positive integer, $T$ a subset of $[a, b]-S$ and, for each integer $n$ greater than $N$ and each $x$ in $T,\left|h_{n}(x)\right|<\epsilon$, then $l_{g} T<l_{0}[a, b]-\delta$.

If $n$ is a positive integer, then there exists a number $x$ in $[a, b]-S$ 
such that for some integer $m$ greater than $n,\left|h_{m}(x)\right| \geqq \epsilon$. Otherwise, the set $[a, b]-S$ is a set $T$ for which the above supposition is violated. For each positive integer $n, U_{n}$ denotes the set such that $x \in U_{n}$ only if $x \in[a, b]-S$ and $\left|h_{m}(x)\right| \geqq \epsilon$ for some integer $m$ greater than $n$. We see that $U_{n+1}$ is a subset of $U_{n}$. We shall prove that there exists a nonempty and closed number set $C_{n}$ such that $C_{n}$ is a subset of $U_{n}$ and $C_{n+1}$ is a subset of $C_{n}$. If $y \in C_{n}$ for $n=1,2,3, \cdots$, then $y$ $\in[a, b]-S$ and $h_{n}(y)$ not $\rightarrow 0$ as $n \rightarrow \infty$, contrary to the hypothesis of the theorem. This contradiction will show that our supposition is false and the theorem will then be established.

If $r_{n}=l_{g} U_{n}$, then $\left\{r_{n}\right\}_{n=1}^{\infty}$ is a nonincreasing sequence with a nonnegative limit $r$. If $r=0$ and $k$ is a positive integer such that $r_{k}<\delta$, the set $[a, b]-\left[S+U_{k}\right]$ is a set $T$ for which our supposition is violated. Consequently, $r>0$.

$H$ denotes the set such that $x \in H$ only if $x$ is $a, b$, or a number in $[a, b]$ where, for some positive integer $n, h_{n}$ is not continuous. If $H$ is finite, $K=H$; if $H$ is infinite, $K$ is a finite subset of $H$ containing $a$ and $b$, such that $\sum_{x \in H-K}[g(x+)-g(x-)]<r \theta$, where $\theta$ is a positive number less than $1 / 4$. If there is a number $y$ such that $y \in K$ and $y \in U_{n}$ for $n=1,2,3, \cdots$, we take $C_{n}=(y)$ for $n=1,2,3, \cdots$.

Suppose there is a positive integer $k$ such that $K$ and $U_{k}$ have no common part. If $n$ is an integer greater than $k, U_{n}$ is not a subset of $H$; $U_{n}^{\prime}$ denotes $U_{n}-H \cdot U_{n} ; Q_{n}$ denotes the set such that $x \in Q_{n}$ if and only if $x$ is in the segment $(a, b)$ and there is an integer $m$ greater than $n$ such that $h_{m}$ is continuous at $x$ and $\left|h_{m}(x)\right| \geqq \epsilon . Q_{n}$ is open, $Q_{n+1} \subset Q_{n}$, and $U_{n}^{\prime}=Q_{n}-(H+S) \cdot Q_{n}$; if $x \in K$ and $x \notin S$, then $x \notin Q_{n}$.

There exists a finite collection $G_{1}$ of mutually exclusive intervals such that if $[p, q] \in G_{1}$ then $g$ is continuous at $p$ and at $q, G_{1}^{*} \subset Q_{k+1}$, and $0 \leqq l_{g} Q_{k+1}-l_{g} G_{1}^{*}<r \theta$, so that $l_{g} G_{1}^{*}>r-r \theta$. If $i$ is a positive integer less than $k+2, C_{i}^{\prime}$ denotes the closed set $G_{1}^{*}$. If $i$ is an integer greater than 1 , then $l_{g}\left(Q_{k+1} \cdot G_{1}^{*}\right) \geqq r-r \theta$.

For each integer $i$ greater than 1 , there exists a finite collection $G_{i}$ of mutually exclusive intervals such that if $[p, q] \in G_{i}$, then $g$ is continuous at $p$ and at $q, G_{i}^{*} \subset Q_{k+1} \cdot G_{i-1}^{*}, 0 \leqq l_{g}\left(Q_{k+i} \cdot G_{i-1}^{*}\right)-l_{g} G_{i}^{*}<r \theta^{i}$ and, for each integer $j$ greater than $i, l_{g}\left(Q_{k+j} \cdot G_{i}^{*}\right) \geqq r-r \theta-r \theta^{2}-\cdots$ $-r \theta^{i}$. For each positive integer $i, C_{k+\imath}^{\prime}$ denotes the closed set $G_{i}^{*}$. Suppose $Q$ is an open set covering $(H+S) \cdot Q_{k}$ such that $l_{\theta} Q<r \theta$. Since $l_{\theta} C_{k+1}^{\prime}>r(1-2 \theta) /(1-\theta)>r \theta$, then $C_{n}^{\prime}$ is not a subset of $Q$ $(n=1,2,3, \cdots)$. If $C_{n}$ is the closed set $C_{n}^{\prime}-C_{n}{ }^{\prime} \cdot Q$, then $U_{n} \supset C_{n}$ $\supset C_{n+1}$, and Theorem $\mathrm{B}$ is established.

4. g-summable functions. In this section we consider functions on an interval $[a, b]$, and suppose the nondecreasing function $g$ is such 
that $g(x)=g(a)$ for $x<a$ and $g(x)=g(b)$ for $x>b$.

TheOREm C. If $S$ is a proper subset of the interval $[a, b], l_{g} S=0$, $\left\{f_{n}\right\}_{n=1}^{\infty}$ a sequence of simple step-functions, uniformly bounded on $[a, b]$, which converges to 0 on $[a, b]-S, f_{n}(x+) \rightarrow 0$ as $n \rightarrow \infty$ if $a \leqq x$ $<b$ and $g(x+)>g(x)$ and $f_{n}(x-) \rightarrow 0$ as $n \rightarrow \infty$ if $a<x \leqq b$ and $g(x)$ $>g(x-)$, then

$$
\int_{a}^{b} f_{n} d g \rightarrow 0 \quad \text { as } n \rightarrow \infty \text {. }
$$

Proof. Suppose $M$ is a number such that if $x \in[a, b]$ and $n$ is a positive integer, then $\left|f_{n}(x)\right|<M$. Suppose $\epsilon$ is a positive number and $\epsilon_{1}=\epsilon /\{4+8[g(b)-g(a)]\}$ and $\delta_{1}=\epsilon / 2 M$.

There exists a subset $T_{1}$ of $[a, b]-S$ and a positive integer $N_{1}$ such that, if $n>N_{1}$ and $x \in T_{1}$, then $\left|f_{n}(x)\right|<\epsilon_{1}$ and $l_{g} T_{1} \geqq l_{g}[a, b]-\left(\delta_{1} / 2\right)$. Suppose $H$ is the set such that $x \in H$ if and only if $x \in[a, b]$ and $g$ is not continuous at $x$ or, for some positive integer $n, f_{n}$ is not continuous at $x$. There exists a finite subset $K$ of $H$ such that

$$
\sum_{x \in H-K}[g(x+)-g(x-)]<\delta_{1} / 2 .
$$

If $T=T_{1}-(H-K) \cdot T_{1}$, then $l_{g} T+l_{g}[a, b]-\delta_{1}$.

There exists a sequence $\left\{h_{n}\right\}_{n=0}^{\infty}$ of nondecreasing simple step-functions such that $h_{n}(a)=g(a), h_{n}(b)=g(b), l_{h_{n}} T \geqq l_{g}[a, b]-\delta_{1}$ or $l_{h_{n}}\{[a$, $b]-T\} \leqq \delta_{1}$ and, for each number $x$ in $[a, b],\left|g(x)-h_{n}(x)\right|<1 / n$.

Now, if each of $m$ and $n$ is a positive integer (cf. (1.1))

$$
\begin{aligned}
\int_{a}^{b} f_{m} d h_{n}= & \sum_{x \in T \cdot(a, b]} \frac{f_{m}(x)+f_{m}(x-)}{2}\left[h_{n}(x)-h_{n}(x-)\right] \\
& +\sum_{x \in T \cdot[a, b)} \frac{f_{m}(x+)+f_{m}(x)}{2}\left[h_{n}(x+)-h_{n}(x)\right]+\sum_{3},
\end{aligned}
$$

where $\left|\sum_{3}\right| \leqq M \delta_{1}=\epsilon / 2, \quad \sum_{3}$ being a sum of like terms taken for $x \in[a, b]-T$. If we consider separately those terms for which $x \in K \cdot T$ and for which $x \in T-K \cdot T$, we see that there exists a positive integer $N$ such that if $m, n>N$, then $\left|\int_{a}^{b} f_{m} d h_{n}\right|<\epsilon$.

Now, $\int_{a}^{b} f_{m} d g=\int_{a}^{b} f_{m} d h_{n}+\int_{a}^{b} f_{m} d\left(g-h_{n}\right)$ so that, if we use integration by parts, and $m, n>N$,

$$
\left|\int_{a}^{b} f_{m} d g\right| \leqq\left|\int_{a}^{b} f_{m} d h_{n}\right|+\left|\int_{a}^{b}\left(g-h_{n}\right) d f_{m}\right| \leqq \epsilon+\frac{1}{n} V_{a}^{b} f_{m}
$$

or $\int_{a}^{b} f_{m} d g \rightarrow 0$ as $m \rightarrow \infty$. 
This completes the proof of Theorem C.

The statement that the function $f$ is g-summable on $[a, b]$ means that there exists a sequence $\left\{f_{m}\right\}_{m=1}^{\infty}$ of simple step-functions, uniformly bounded on $[a, b]$, such that $f_{m}(x) \rightarrow f(x)$ as $m \rightarrow \infty$ for every number $x$ in $[a, b]$ or in $[a, b]-S$, where $S$ is a subset of $[a, b]$ of outer $g$-length 0 , and $f_{m}(x-) \rightarrow f(x-)$ as $m \rightarrow \infty$ if $a<x \leqq b$ and $g(x)$ $>g(x-)$, and $f_{m}(x+) \rightarrow f(x+)$ as $m \rightarrow \infty$ if $a \leqq x<b$ and $g(x+)$ $>g(x)$.

We see by Theorem $\mathrm{C}$ that if $f$ is $g$-summable on $[a, b]$, then there exists a number $J$ such that if $\left\{f_{m}\right\}_{m=1}^{\infty}$ is any sequence of simple step-functions having the above properties:

$$
\int_{a}^{b} f_{m} d g \rightarrow J
$$

as $m \rightarrow \infty$.

We define the number $J$ to be the integral $\int_{a}^{b} f d g$ on $[a, b]$ of $f$ with respect to $g$.

It is easy to show that if $\left\{f_{m}\right\}_{m=1}^{\infty}$ is a uniformly bounded sequence of $g$-summable functions converging in the manner described in the above definition to a function $f$, then $f$ is $g$-summable and $\int_{a}^{b} f_{m} d g$ $\rightarrow \int_{a}^{b} f d g$ as $m \rightarrow \infty$.

Remark added in proof. My attention has been called to the fact that the definition I accredited to Lane was given by H. L. Smith, On the existence of the Stieltjes integral (Trans. Amer. Math. Soc. vol. 27 (1925) pp. 491-495).

\section{BIBLIOGRAPHY}

1. D.-Th. Egoroff, Sur les suites de fonctions mesurables, C.R. Acad. Sci. Paris vol. 152 (1911) pp. 244-246.

2. R. E. Lane, The integral of a function with respect to a function, Proc. Amer. Math. Soc. vol. 5 (1954) pp. 59-66.

3. F. Reisz, Sur l'intégrale de Lebesgue, Acta Math. vol. 42 (1920) pp. 191-205

De Paul University 